\title{
TERAPIA FARMACOLÓGICA DOS SINTOMAS MOTORES NA DOENÇA DE
} PARKINSON: LEVODOPA

\section{PHARMACOLOGICAL THERAPY FOR MOTOR SYMPTOMS OF PARKINSON'S DESEASE: LEVODOPA}

\author{
Raquel de Oliveira Vilhena ${ }^{1}$; Marco Andre Cardoso ${ }^{2}$; Roberto Pontarolo ${ }^{1}$
}

\author{
${ }^{1}$ Centro de Estudos em Biofarmácia, Departamento de Farmácia, Universidade Federal do \\ Paraná, Curitiba-Paraná \\ ${ }^{2}$ Instituto de Pesquisa Pelé Pequeno-Príncipe, Curitiba-Paraná
}

\section{RESUMO:}

A doença de Parkinson é uma das afeç̧ões neurodegenerativas mais comuns na população com mais de 65 anos. A principal característica patológica dessa doença é a perda progressiva de neurônios dopaminérgicos da substância negra compacta, resultando na depressão estriatal de dopamina e, assim, o aparecimento dos sintomas motores: tremor de repouso, bradicinesia, rigidez e instabilidade postural. Nos últimos anos, diversos medicamentos surgiram para o tratamento dos sintomas motores. Entretanto, desde a introdução da terapia por suplementação de dopamina, a levodopa é considerada padrão ouro. Devido à alta metabolização periférica, é comum a associação da levodopa a inibidores enzimáticos, como a carbidopa e entacapona. Apesar da boa resposta obtida utilizando a levodopa, é comum o aparecimento de sintomas como discinesias e flutuações ao longo do tratamento, os quais parecem estar relacionados ao uso prolongado desse fármaco e às altas doses administradas. Por isso, é necessário o uso de estratégias nessa terapia medicamentosa, evitando-se a ocorrência desses sintomas.

Palavras-chave: levodopa, doença de Parkinson, sintomas motores.

\section{ABSTRACT:}

Parkinson's disease is the most commom neurodegenerative disorder in population over 65 years old. The main pathological feature of this disease is the progressive loss of dopaminergic neurons of the substantia nigra compacta, resulting in depression of striatal dopamine and thus the onset of motor symptoms: tremor, bradykinesia , rigidity and postural instability. In recent years, various drugs have emerged for the treatment of motor symptoms. However, since the introduction of dopamine-replacement therapy, levodopa is considered the gold standard. Because of the high peripheral metabolism of this drug, is common to the combination of levodopa with enzymatic inhibitors such as carbidopa and entacapone. Despite the good response obtained using levodopa is common the appearance of neural symptoms such as dyskinesia and fluctuations, which seem to be related to the prolonged use of this drug and high dose administration. Therefore, the use of strategies in drug therapy is necessary to avoid the occurrence of these symptoms.

Keywords: Parkinson's disease, levodopa, motor symptoms 


\section{DOENÇA DE PARKINSON}

\subsection{Histórico e etiologia da doença de Parkinson}

Em 1817, James Parkinson descreveu as principais características clínicas da doença de Parkinson (DP) e seu agravamento (GOOLE; AMIGHI, 2009). Passado mais de cem anos foi, então, que se descobriu que a característica central da doença era a perda de neurônios dopaminérgicos na substância nigra compacta. Em 1958, com a descoberta de dopamina no cérebro de mamíferos, houve um aceleramento nas pesquisas. Descobriu-se nesse período que a perda de neurônios dopaminérgicos da substância negra compacta levava a deficiência de dopamina estriatal (responsável pela maioria dos sintomas da doença) e o tratamento com precursor da dopamina (levodopa) aliviava a maioria dos sintomas (DAUER; PRZEDBORSKI, 2003).

ADP é uma doença neurodegenerativa, crônica, progressiva e multifatorial que se manifesta geralmente próximo aos 55 anos, sendo que sua incidência aumenta conforme o aumento da idade. Os casos esporádicos da DP, ou seja, os aparentemente não relacionados a fatores genéticos correspondem a $95 \%$ e os $5 \%$ restantes são os casos relacionados à hereditariedade (DAUER; PRZEDBORSKI, 2003; WEINTRAUB; COMELLA; HORN, 2008).

Pacientes com DP sofrem com sintomas motores (tremor de repouso, bradicinesia, rigidez e instabilidade postural) e também sintomas não-motores (depressão, diminuição do sentido olfativo e distúrbios do sono) (KHOR; HSU, 2007). $\mathrm{Na}$ ausência de tratamento, o Parkinson progride num período de 5 a 10 anos, levando a um estágio de rigidez, onde o paciente não consegue mais cuidar de si mesmo. A rigidez muscular pode gerar complicações como, por exemplo, pneumonia por aspiração e embolia pulmonar, podendo levar o paciente à morte (STANDAERT; YOUNG, 2007).

\subsection{Fisiopatologia da doença de Parkinson}

O estriado juntamente com as outras regiões que compõem o gânglio basal o cérebro é responsável por modular o fluxo de informações recebidas pelo córtex cerebral para os neurônios motores da coluna espinhal. O estriado é composto por neurônios que exibem predominantemente receptores para dopamina. A inervação dopaminérgica que regula as atividades nessa região é fornecida por outra região cerebral conhecida como substância negra compacta. A dopamina é uma catecolamina sintetizada nos terminais dos neurônios dopaminérgicos a partir da tirosina por uma sequência de ações da tirosina hidrolase e AADC. Nos indivíduos acometidos pela DP, a degeneração dos neurônios dopaminérgos (70-80\% de perda neuronal) na 
substância negra compacta leva a deficiência de dopamina no estriado, afetando os estímulos que controlam o movimento (STANDAERT; YOUNG, 2007).

A causa da degeneração dos neurônios dopaminérgicos ainda não é clara, mas a combinação de fatores genéticos e ambientais parece estar relacionada (REZAK, 2007). Fatores secundários como certas infecções neurológicas e medicamentos também podem causar a morte desses neurônios, resultando em formas secundárias da doença (WEINTRAUB; COMELLA; HORN, 2008). Qualquer doença que inclua deficiência de dopamina estriatal ou dano estriatal direto pode levar à síndrome conhecida como parkinsonismo. O parkinsonismo é caracterizado por tremor de repouso, rigidez, lentidão ou ausência de movimentos voluntários, instabilidade postural e incapacidade de iniciar movimentos voluntários. ADP é a causa mais comum dessa síndrome, sendo responsável por $80 \%$ dos casos (DAUER; PRZEDBORSKI, 2003).

\subsection{Diagnóstico da doença de Parkinson}

A avaliação clínica é a principal ferramenta para o diagnóstico da DP. O paciente deve apresentar pelo menos dois dos sintomas motores primários da doença: bradicinesia, rigidez muscular, instabilidade postural e tremor. Sintomas como demência, problemas na motilidade gastrointestinal, avaliação da resposta à terapia com levodopa, micrografia, perda de expressão facial e características não-motoras como distúrbios afetivos e do sono, comprometimento cognitivo e queixas sensoriais também são consideradas como componentes do diagnóstico da DP. (NUSSBAUM; POLYMEROPOULOS, 1997; REZAK, 2007).

Na avaliação neuropatológica, a perda de células e a presença de corpúsculos de Lewis na substância negra são requeridas para o diagnóstico. (SPILLANTINI et al., 1998).

\subsection{Tratamento da doença de Parkinson}

Nos últimos anos, as opções para o tratamento da doença de Parkinson se ampliaram tanto no enfoque farmacoterapêutico quanto neurocirúrgico. Dentre os medicamentos disponíveis estão os agonistas de dopamina, anticolinérgicos, inibidores da monoaminoxidase-B e catecol-O-metiltransferases, amantadina e levodopa. Os avanços na terapêutica da DP têm proporcionado um melhora significativa na qualidade de vida dos pacientes (REZAK, 2007). 


\section{LEVODOPA}

Desde a introdução da terapia por suplementação de dopamina para tratamento dos sintomas motores da DP, a levodopa tem sido considerada padrão ouro (CLARKE; GUTTMAN, 2002; GONÇALVES et al., 2012). Esse medicamento é eficaz no tratamento dos sintomas da DP, obtendo-se uma boa resposta dessa terapia, mesmo nos estágios avançados dessa doença (GOOLE; AMIGHI, 2009).

Ao contrário da dopamina, a levodopa é capaz de atravessar a barreira hematoencefálica. No tecido cerebral, a levodopa é convertida através da ação da enzima L-aminoácido aromático descarboxilase (AADC) em seu metabólito ativo dopamina. A dopamina é então armazenada nas vesículas pré-sinápticas até sua liberação para os receptores pós-sinápticos (GOOLE; AMIGHI, 2009; LEWITT, 2009).

Apesar de ser bem absorvida após administração oral através do transporte saturável de aminoácidos, a levodopa apresenta baixa biodisponibilidade devido à alta metabolização periférica. Quando administrada isoladamente, apenas $1 \%$ do fármaco inalterado consegue alcançar a circulação cerebral (KHOR; HSU, 2007; GONÇALVES et al., 2012).

As enzimas responsáveis pela metabolização periférica da levodopa são a catecol-O-metiltransferase (COMT), convertendo-a em 3-O-metildopa, e a AADC, convertendo-a em dopamina (KHOR; HSU, 2007). Essa dopamina, na região periférica, terá ação nos receptores dopaminérgicos, provocando reações adversas como náuseas, hipotensão ortostática e sudorese.

Por isso, a levodopa é administrada associada a inibidores enzimáticos como carbidopa e benserazida (inibidores da AADC) e tolcapona e entacapona (inibidores da COMT). Essa associação auxilia na redução da conversão da levodopa em dopamina, reduzindo as reações adversas e aumentando a quantidade de levodopa plasmática disponível para atravessar a barreira hematoencefálica (GOOLE; AMIGHI, 2009).

Os pacientes em tratamento com levodopa também podem apresentar efeitos gerados no sistema nervoso central, como sedação, sintomas psiquiátricos, flutuações motoras e discinesias. O aparecimento desses sintomas está relacionado à alta variabilidade inter e intraindividual, ou seja, variações na absorção e metabolização desse fármaco (GORDIN; KAAKKOLA; TERÄVÄINEN, 2004; REZAK, 2007).

As flutuações motoras e as discinesias são sintomas motores que influenciam diretamente na qualidade de vida dos pacientes. As flutuações motoras podem variar desde as mais simples como o fenômeno "wearing off", observados entre os intervalos de dose, até oscilações graves na função motora, como o fenômeno "on-off'. Nesse fenômeno o paciente alterna entre manifestações graves de sintomas parkinsonianos (efeito "off') e estado motor quase normal (efeito "on") seguido de movimentos involuntários (REZAK, 2007). 
Durante a evolução da DP, é comum o paciente apresentar perda ou ganho de peso. Esse fator influencia na concentração plasmática da levodopa e consequentemente na ação cerebral desse medicamento. Quanto maior o peso do paciente, menor a concentração plasmática observada do fármaco. Provavelmente, isso ocorre devido ao aumento do volume de distribuição (MÜLLER et al., 2000).

Além disso, as discinesias e flutuações parecem estar relacionadas ao uso prolongado da levodopa e também às altas doses administradas desse fármaco. Mudanças no perfil farmacocinético de pacientes em tratamento com a levodopa por mais de cinco anos foram observados em um estudo realizado por Murata (2006). Essas alterações podem ter sido causadas pelo aumento da absorção desse medicamento. Também, com a progressão da doença, a degeneração dos neurônios dopaminérgicos da substância negra compacta e subsequente redução da dopamina cerebral, os terminais dopaminérgicos não são mais capazes de exercer efeitos compensatórios sob a levodopa exógena e a duração da resposta à levodopa é reduzida. Nesse estágio, os pacientes se tornam muito sensíveis às variações da concentração de levodopa plasmática, apresentando flutuações motoras e discinesias (GOOLE; AMIGHI, 2009).

Em formulações de liberação imediata, devido ao curto tempo de meia-vida da levodopa, os receptores dopaminérgicos são estimulados de forma anormal e descontínua, resultando em oscilações da resposta clínica desse medicamento. Estudos mostraram que quando os pacientes eram tratados com a forma farmacêutica líquida, as concentrações plasmáticas de levodopa se mantiveram mais estáveis. Essa estabilidade está provavelmente relacionada à possibilidade de ajustes individuais da dose, permitindo ao paciente receber a dose de acordo com sua necessidade. Já as formulações de liberação controlada permitem a liberação de modo contínuo num período prolongado de tempo, tendo como vantagem a manutenção das concentrações plasmáticas de levodopa, permitindo a redução de tomadas ao longo do dia e, assim, uma melhora na adesão ao tratamento pelo paciente (GOOLE; AMIGHI, 2009). No entanto, há algumas limitações para esse tipo de formulação, como a região de absorção do fármaco (onde há a presença do transporte facilitado para aminoácidos neutros) e a influencia de aminoácidos provenientes da alimentação, os quais competirão com a levodopa pelo sistema de transporte (LEWITT, 2009).

Visando a redução dessas complicações e uma melhora na qualidade de vida do paciente, a escolha da terapia na DP deve ter como objetivo maximizar as possíveis estratégias poupadoras de levodopa. No entanto, em casos que essas estratégias deixam de controlar os sintomas ou quando o paciente está exposto a situações de perigo devido aos sintomas, a terapia com levodopa faz-se necessária (REZAK, 2007).

A levodopa quando administrada com um inibidor da AADC (carbidopa ou benserazida) tem sua via de metabolização pela enzima COMT ativada, pois assim 
como a dopamina, a levodopa possui um grupo catecol que pode ser inativado por essa enzima. Isso propicia um aumento na variação da concentração plasmática do fármaco. Então, com a finalidade de suavizar as flutuações plasmáticas apresentadas pelos pacientes, sugere-se a associação de um inibidor da COMT, como a entacapona (MURATA, 2006).

A principal ação terapêutica dos inibidores da COMT é bloquear a conversão da levodopa em 3-O-metildopa, aumentando o tempo de meia vida no plasma assim como a fração da dose que chega ao cérebro. A diferença entre esses fármacos é que a tolcapona parece ter ação cerebral e periférica, apresentando longa duração e sendo normalmente administrada duas ou três vezes ao dia. Já a entacapona tem ação de curta duração (cerca de $2 \mathrm{~h}$ ) e sua ação é periférica, sendo administrada juntamente com cada dose de levodopa associada à carbidopa ou benserazida (GONÇALVES et al., 2012).

\section{CONCLUSÃO}

Diversos são os fatores que influenciam na concentração plasmática da levodopa e consequentemente na ação cerebral desse medicamento. Com a progressão da doença os pacientes passam a apresentar fenômenos como as discinesias e flutuações, as quais impactam diretamente na qualidade de vida desses pacientes. Além disso, o uso prolongado da levodopa e também a administração de altas doses desse medicamento parecem estar relacionados ao aparecimento desses fenômenos.

Atualmente buscam-se estratégias para aumentar a disponibilidade da levodopa. Para isso, as pesquisas se concentram em modular ou desenvolver fórmulas capazes de fornecer e sustentar um fluxo constante de levodopa, prolongando sua resposta. Os estudos também se voltam ao desenvolvimento de inibidores enzimáticos que sejam mais seguros e eficazes (ROCHA et al., 2013).

\section{REFERÊNCIAS}

CLARKE, C. E.; GUTTMAN, M. Dopamine agonist monotherapy in Parkinson's disease. The Lancet, v. 360, n. 9347, p.1767-1769, 2002.

DAUER, W.; PRZEDBORSKI, S. Parkinson's Disease: Mechanisms and Models. Neuron, v. 39, n. 6, p.889-909, 2003.

GONÇALVES, D. et al. Bioanalytical chromatographic methods for the determination of catechol-O-methyltransferase inhibitors in rodents and human samples: A review. 
Analytica Chimica Acta, v. 710, n. 0, p.17-32, 2012.

GOOLE, J.; AMIGHI, K. Levodopa delivery systems for the treatment of Parkinson's disease: An overview. International Journal of Pharmaceutics, v. 380, n. 1-2, p.1-15, 2009.

GORDIN, A.; KAAKKOLA, S.; TERÄVÄINEN, H. Clinical advantages of COMT inhibition with entacapone - a review. Journal of Neural Transmission, v. 111, n. 10-11, p.1343-63, 2004.

KHOR, S. P.; HSU, A. The pharmacokinetics and pharmacodynamics of levodopa in the treatment of Parkinson's disease. Current Clinical Pharmacology, v. 2, n. 3, p.234-43, 2007.

LEWITT, P. A. Levodopa therapeutics for Parkinson's disease: new developments. Parkinsonism \& Related Disorders, v. 15, Supplement 1, n. 0, p.S31-S34, 2009.

MÜLLER, T. et al. Levodopa in plasma correlates with body weight of parkinsonian patients. Parkinsonism \& Related Disorders, v. 6, n. 3, p.171-173, 2000.

MURATA, M. Pharmacokinetics of L-dopa. Journal of Neurology, v. 253, n. suppl. 3, p.47-52, 2006.

NUSSBAUM, R. L.; POLYMEROPOULOS, M. H. Genetics of Parkinson's disease. Human Molecular Genetics, v. 6, n. 10, p.1687-91, 1997.

REZAK, M. Current pharmacotherapeutic treatment options in Parkinson's disease. Disease-a-Month, v. 53, n. 4, p.214-22, 2007.

ROCHA, J. F. et al. Effect of opicapone and entacapone on levodopa pharmacokinetics when administered with immediate release $100 / 25 \mathrm{mg}$ levodopa/carbidopa in healthy subjects. Journal of the Neurological Sciences, v. 333, Supplement 1, n. 0, p.115116, 2013.

SPILLANTINI, M. G. et al. alpha-Synuclein in filamentous inclusions of Lewy bodies from Parkinson's disease and dementia with lewy bodies. Proceedings of the National Academy of Sciences v. 95, n. 11, p.6469-73, 1998.

STANDAERT, D. G.; YOUNG, A. B. Tratamento dos Distúrbios Degenerativos do 
Sistema Nervoso Central. In: BRUNTON, L. L., LAZO, J. S. e PARKER, K. L. (Ed.). As Bases Farmacológicas da Terapêuticaed. S.I: Mc Graw-Hill Companies, 2007. p. 469-486.

WEINTRAUB, D.; COMELLA, C. L.; HORN, S. Parkinson's Disease-Part 1: Pathophysiology, Symptoms,Burden, Diagnosis, and Assessment. The American Journal of Manager Care, v. 14, n. 2, p.S40-S48, 2008. 